\title{
Tristetraprolin: a novel mediator of the anticancer properties of resveratrol
}

\author{
C. Li, C. Tang and G. He \\ Department of General Surgery, The First Affiliated Hospital, \\ University of South China, HengYang, Hunan \\ Corresponding author: G. He \\ E-mail: hegengsh@163.com \\ Genet. Mol. Res. 15 (2): gmr.15027213 \\ Received August 14, 2015 \\ Accepted January 13, 2016 \\ Published May 20, 2016 \\ DOI http://dx.doi.org/10.4238/gmr.15027213
}

\begin{abstract}
Resveratrol is a natural compound that exhibits anticancer properties. Previous studies have proved that it can inhibit the proliferation of breast cancer cell lines and upregulate some cytokines such as cyclooxygenase-2 (COX-2) and vascular endothelial growth factor (VEGF). The initiation and progression of cancer are associated with the abnormal expression of multiple cytokines. Tristetraprolin (TTP), an mRNA-binding protein, is one of the key proteins that participate in regulating cytokine expression. Two different proliferation assays on MCF-7 cells showed that the cell proliferation rate significantly reduced following treatment with resveratrol. Most importantly, we found that resveratrol promoted TTP expression at both the mRNA and protein level in a dose- and time-dependent manner. In addition, the expression of COX-2 and VEGF were significantly suppressed by resveratrol while that of inducible nitric oxide synthase (iNOS) was upregulated. Lastly, the effects of resveratrol on both MCF-7 proliferation and expression of COX-2, VEGF, and iNOS were significantly inhibited by TTP knockdown, indicating that TTP mediates the anticancer properties of resveratrol. In summary, we conclude that resveratrol inhibits the proliferation of MCF-7 cells by
\end{abstract}


TTP upregulation, which is associated with downregulation of COX-2 and VEGF and upregulation of iNOS.

Key words: Breast cancer; Resveratrol; Tristetraprolin; Proliferation

\section{INTRODUCTION}

Cancer incidence and mortality are on an upward trend and prevention and treatment have become urgent health issues. Disorderly growth and high metastatic and proliferative activities are important features of malignant tumor cells (Hong and Zeng, 2012). The Weinberg research group has shown that a variety of proteins closely related to tumor cell differentiation, apoptosis, and invasion and thus involved in the development of malignant tumors, are disorderly expressed in tumor cells (Hanahan and Weinberg, 2011). Protein expression is closely related to intracellular levels of messenger RNA (mRNA), and regulating the mRNA levels is one of the key steps in controlling protein expression. Recent studies have determined that disorderly mRNA degradation is involved in the overexpression of the genes related to inflammation, growth, and malignant tumors, suggesting that regulation of mRNA degradation may be a potential target for cancer prevention and treatment (Maquat, 2005).

mRNA degradation involves many mRNA-binding proteins, which regulate the stabilization or degradation of mRNA by binding to AU-rich elements (AREs) in the 3' untranslated region of mRNAs and recruiting cofactors (Melanson et al., 2011). The most common mRNA-binding protein include AUF1, HuR, TTP, etc. (Maquat, 2005). AUF1 shuttles between the nucleus and cytoplasm, regulating the degradation of target mRNAs including P21 and cyclin D1 (CD1) (Shchors et al., 2002). HuR, also known as embryonic lethal abnormal vision RNA-binding protein, inhibits mRNA degradation of vascular endothelial growth factor (VEGF), cyclooxygenase (COX)-2, and cyclin A (Young et al., 2012). Tristetraprolin (TTP), a zinc finger protein family member rich in proline and serine amino acids with multiple PPPP repeats, contains two Cys-Cys-Cys-His zinc finger structures that are responsible for mRNA binding and degradation (Patino et al., 2006).

TTP promotes mRNA degradation by binding to AREs of a variety of mRNA molecules (Ogilvie et al., 2005). Under normal circumstances, TTP is expressed in a variety of cells, participating in the regulation of growth factors, inflammatory cytokines, and chemokines (Qiu et al., 2012). The expression, phosphorylation, and subcellular localization of TTP, together with interactions of TTP with other proteins, are closely related to the occurrence and development of malignant tumors, suggesting that TTP expression and dysfunction are an important indicator of cancer development (Sanduja et al., 2012). The expression of TTP can be induced by many factors, including insulin (Cao et al., 2008), mitogen (Hitti et al., 2006), apolipoprotein (Yin et al., 2011), and green tea (Cao et al., 2007). The upregulation of TTP has been found to inhibit the development of inflammation, atherosclerosis, and some malignancies (Yin et al., 2009).

Various cytokines, such as VEGF, and cytokine-producing enzymes, such as COX2, play key roles in the cell proliferation of malignant tumors (Falandry et al., 2011). TTP has been found to downregulate COX-2 and VEGF expression in colon cancers (Lee et al., 2010; Cha et al., 2011). Inducible nitric oxide synthase (iNOS) is an enzyme that can promote endogenous NO generation, the role of which needs to be defined. Nevertheless, studies have found that high concentrations of NO can inhibit the proliferation of tumor cells by blocking 
DNA synthesis and mitochondrial oxidation, thus exerting anti-tumor effects (Harada et al., 2004). Low concentrations of NO can be converted to peroxynitrite, causing damage to DNA and promoting tumor progression and metastasis (Takahashi et al., 2008). Moreover, the Kleinert group has demonstrated that overexpression of TTP can significantly increase the expression of iNOS in human colon cancer cell line DLD-1 (Fechir et al., 2005).

In women, breast cancer is one of the most common malignant tumor types. Currently, the main treatments for breast cancer include chemotherapy, radiotherapy, and surgery, resulting in extremely painful side effects. Therefore, finding less toxic, safer, and more effective anticancer drugs is the current research focus (Tan et al., 2008). Resveratrol, a natural polyphenolic compound present in many plants, can extend life and inhibit inflammation and oxidation (Subramanian et al., 2010). More interestingly, several research groups have proven the prominent anti-cancer effects of resveratrol. It has been found that resveratrol can inhibit the expression of VEGF, thereby inhibiting the development of malignant hepatoma (Zhang et al., 2005). In addition, Degner et al. (2007) showed that resveratrol can inhibit the expression of COX-2 in MCF-7 cells, thus inhibiting the proliferation of breast cancer cells. However, the mechanism of the anti-cancer effects of resveratrol requires clarification. Studying the mechanism will strengthen our understanding of the experimental and theoretical basis for clinical application of the class of natural compounds like resveratrol (Bishayee, 2009).

Here, we first examined TTP expression in different breast cancer types. Using the MCF-7 and MDA-MB-231 breast cancer cell lines as the model, we next investigated the effects of resveratrol on cell proliferation, TTP expression, and the expression of effectors downstream of TTP. Lastly, we examined whether TTP is the mediator for these effects of resveratrol on breast cancer cells by knocking down TTP expression with RNA interference. This study increases the understanding of the anti-cancer effects of resveratrol and the molecular basis for further applications of resveratrol in clinical cancer therapy.

\section{MATERIAL AND METHODS}

\section{Tumor sample collection}

We collected tumor samples from 87 individuals who were suspected to have breast cancers and were admitted to the First Affiliated Hospital of University of South China between May of 2010 and 2012. The pathological analyses showed that 36 samples were ductal carcinoma in situ (DCIS) while the rest was invasive ductal carcinoma (IDC). Samples of normal pericarcinous tissues (12) were collected as the controls. The patient age ranged from 24 to 78 years, with a median age of 46 years. Not all patients received preoperative radiotherapy or chemotherapy before surgeries. Each specimen was split into three parts for RNA and protein extraction and $10 \%$ formalin fixation.

\section{Immunohistochemical analysis}

The fixation, paraffin embedding, sectioning, and staining of tissue specimens were carried out using the routine method. Thin $(3-4 \mu \mathrm{m})$ sections were prepared from formalinfixed, paraffin-embedded tissues and were used for immunocytochemistry. TTP was detected with mouse anti-human antibody (Abcam, Cambridge, MA, USA) used at a 1:200 dilution. Bound antibody was visualized with a biotinylated secondary antibody (Poshide, Wuhan, 
China) and 3,3'-diaminobenzidine (Maixin, Fuzhou, China). Photography was performed using an inverted Olympus microscope. Photographs were analyzed using Wuhan Qianping imaging technology HMIAS2000. The positive signal had a color of light yellow to brown. The gray color intensity of positive cells was used as the indicator of signal strength: the higher the gray value, the lower the signal, and vice versa. We randomly selected five nonoverlapping high power fields and calculated the percentage of positive cells. We classified $\leq 5 \%$ as negative and $>5 \%$ as positive.

\section{Cell culture}

Human breast cancer cell line MCF-7 and MDA-MB-231 were cultured with Dulbecco's Modified Eagle's medium (DMEM) containing 5.6 mM glucose, $1 \mathrm{mM}$ sodium pyruvate, $10 \%$ inactivated fetal bovine serum (FBS), and $100 \mathrm{U} / \mathrm{mL}$ penicillin and streptomycin and incubated at $37^{\circ} \mathrm{C}$ with $5 \% \mathrm{CO}_{2}$. Following a trypan blue exclusion assay of cell viability, cells passing the test $(\geq 95 \%)$ were used for further experiments.

\section{Real-time quantitative RT-PCR (qRT-PCR)}

Total RNA samples were extracted from tissues or cells using TRIzol reagent (Life Technologies, Grand Island, NY, USA). cDNA samples were synthesized using a ReverAid ${ }^{\mathrm{TM}}$ first strand cDNA synthesis kit (Promega, Madison, WI, USA) and used for quantitative PCR (qPCR). Primer sequences were as follows. TTP: 5'-TCGAAGAGACCCTAACCAGGC-3'/ 5'-GCGTAGTCATCAGGATCGGA-3'. AUF1: 5'-GCCTTTCTCCAGATACACCTGAAG-3'/ 5'-CTTATTGGTCTTGTTGTCCATGGG-3'. HuR: 5'-TTCACATCCGATTCAGCC-3'/5'-TC TACTGCCATCATTACACG-3'. COX-2: 5'-TCCTCCTGGAACATGGACTC-3'/5'-GCTCG GCTTCCAGTATTGAG-3'. $\beta$-actin: 5'-TCACCATCTTCCAGGAGCGAG-3'/5'-TGTCGCT GTTGAAGTCAGAG-3'. Three biological replicates were performed for each gene.

\section{Western blot}

Tissue or cell samples were lysed on ice with RIPA buffer and then centrifuged at $10,000 \mathrm{~g}$ at $4^{\circ} \mathrm{C}$ for $10 \mathrm{~min}$. The supernatant was saved and an aliquot was used for sodium dodecyl sulfate polyacrylamide gel electrophoresis (SDS-PAGE). Anti-TTP (Abcam) was used at 1:1000 dilution. The signals were quantified using the Labworks image software (UVP, Upland, CA, USA).

\section{Enzyme-linked immunosorbent assay (ELISA)}

Cells were grown in complete DMEM (containing 10\% FBS) in triplicate for 2 days until they were $80-90 \%$ confluent. The growth medium was removed, and cells were briefly washed with cell medium without FBS. Then, equal volumes of fresh medium containing $2 \%$ FBS were added and cells were allowed to grow for $24 \mathrm{~h}$. The medium was harvested and tested using ELISA kits for COX-2, VEGF, or iNOS (R\&D Systems, MN, USA). Results are reported as $\mathrm{pg} / \mathrm{mL}$ of growth medium. This experiment was performed twice. The final values of VEGF concentration shown were subtracted values from the control (i.e., cell medium containing $2 \%$ FBS). 


\section{MTT assay}

We performed an MTT cell proliferation assay using the method reported by Ahmad et al. (2006). Briefly, MCF-7 and MDA-MB-231 cells were seeded on a 96-well plate at about $1 \times 10^{4} /$ well, incubated with $20 \mu \mathrm{L} 5 \mathrm{mg} / \mathrm{mL}$ MTT for $3 \mathrm{~h}$, washed, and covered with 150 $\mu \mathrm{L}$ dimethyl sulfoxide (DMSO). The optical density (OD) values were then measured on a microplate reader. We examined the cell proliferation rate from day 1 to 7 .

\section{Flow cytometry}

We performed flow cytometry to examine cell proliferation. Briefly, MCF-7 cells were seeded on 6-well plates (about $2 \times 10^{5}$ cells/well), incubated with serum-free DMEM for $24 \mathrm{~h}$, and then treated with 10,20 , or $40 \mu \mathrm{M}$ resveratrol for $6 \mathrm{~h}$. Trypsinized cells were collected, resuspended in $1 \%$ binding buffer at about $1 \times 10^{6} / \mathrm{mL}$, incubated with $30 \mu \mathrm{M}$ bromodeoxyuridine (BrdU) for $40 \mathrm{~min}$, fixed in ethanol, treated with $\mathrm{HCl}$, and neutralized with borax. The cell suspension $(100 \mu \mathrm{L})$ together with $5 \mu \mathrm{L}$ FITC-IgG were added to a 5-mL flow test tube, mixed, incubated in the dark at $22^{\circ} \mathrm{C}$ for $15 \mathrm{~min}$, and run on a flow cytometry instrument after being mixed with $400 \mu \mathrm{L} 1 \mathrm{X}$ binding buffer.

\section{RNA interference}

We conducted the RNA interference experiments using the method described by Joe et al. (2011). TTP siRNA, 5'-UCGCCACC CCAAGUA CAAATT-3' (cis) and 5'-CUCUGCCACAAGUUCUACCTT-3' (trans), as well as the control siRNA, which is not homologous with any known human genes, were synthesized by Shanghai Biological Engineering company. Lipofectamine 2000 (Life Technologies, Grand Island, NY, USA) was used to transfect siRNA into cells. mRNA or protein changes were examined $48 \mathrm{~h}$ following transfection.

\section{Measurement of COX-2 mRNA degradation kinetics}

We followed the method used to measure COX-2 mRNA degradation kinetics in MCF7 and MDA-MB-231 cells. Briefly, we treated the cells with the combination of siRNA and resveratrol (or DMSO) for $3 \mathrm{~h}$, added actinomycin D to terminate mRNA transcription, and then ran qRT-PCR at different time points $(0,40,80$, and $160 \mathrm{~min})$ to examine the expression levels of COX-2 mRNA.

\section{Statistical analysis}

We used SPSS 12.0 to perform the statistical analyses. Two groups were compared using the Student $t$-test. Statistical significance was considered $\mathrm{P}<0.05$.

\section{RESULTS}

TTP expression is significantly inhibited in human breast cancers. TTP expression and function are inversely related to tumorigenesis and progression of malignant tumors (Sanduja et al., 2012). The upregulation of TTP has been found to inhibit the development of 
malignant tumors. To determine whether dysregulated TTP expression contributes to breast tumor development in the Chinese population, we compared levels of TTP, AUF1, and HuR mRNAs between breast tumors and normal pericarcinous tissues of 87 patients. The results showed that compared to that in normal tissues, TTP mRNA expression in breast cancer tissues significantly decreased, while the expression of AUF1 and HuR mRNA did not change significantly (Figure 1A-C).

A

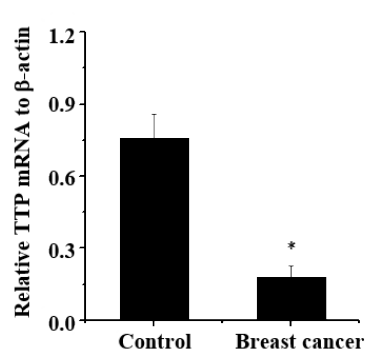

D
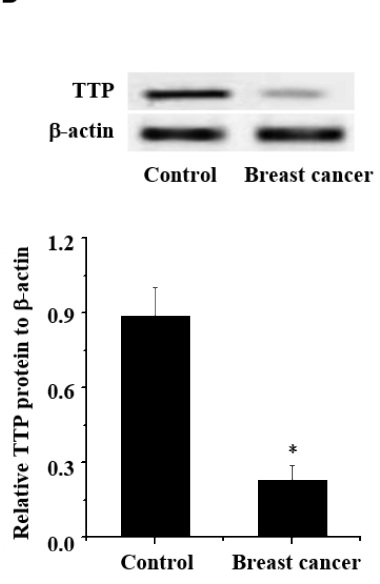

B

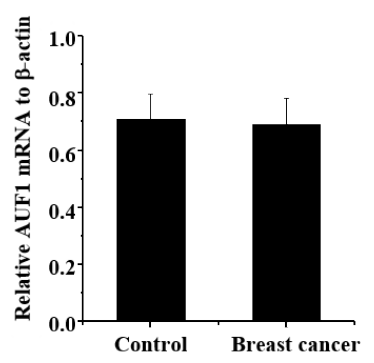

E
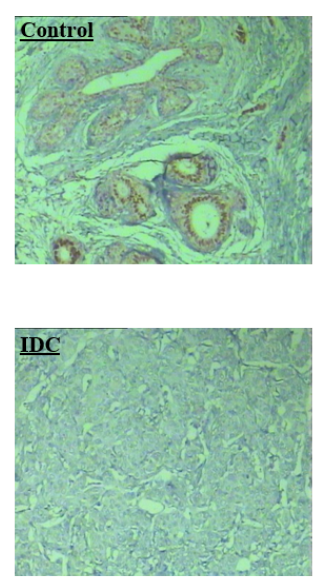

C
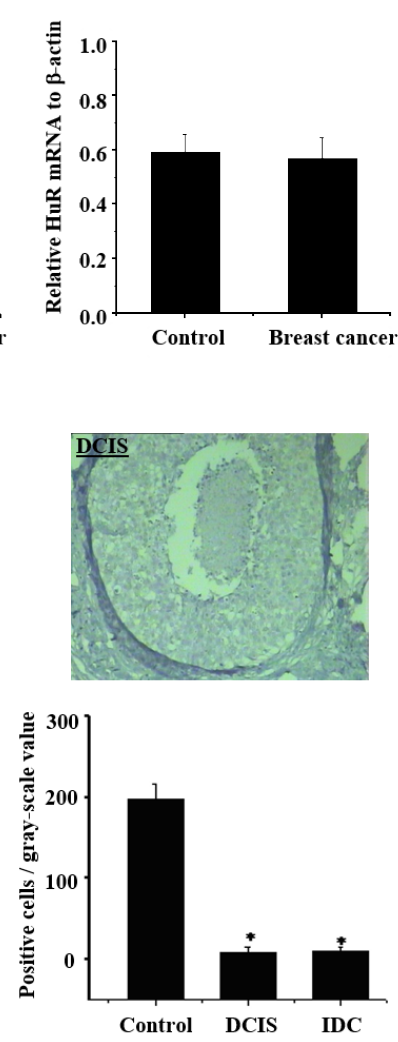

Figure 1. TTP mRNA and protein expression levels in human breast cancers. mRNA levels were measured by qRT-PCR. TTP protein levels were measured by western blotting and immnocytochemistry. A. TTP mRNA expression levels in breast cancer tissues $v s$ that in controls $(\mathrm{N}=3) . * \mathrm{P}<0.05$. B. AUF1 mRNA expression levels in breast cancer tissues $v s$ that in controls $(\mathrm{N}=3)$. C. HuR mRNA expression levels in breast cancer tissues $v s$ that in controls $(\mathrm{N}=3)$. D. TTP protein levels in breast cancer tissues $v s$ that in controls $(\mathrm{N}=3) . * \mathrm{P}<0.05$. E. Immunohistochemical detection and quantification of TTP in DCIS and IDC tumor tissues and pericarcinous normal tissues. Magnification: 400X. $* \mathrm{P}<0.05$.

To investigate whether protein expression of TTP is also repressed in tumor tissues relative to normal tissues, we performed western blotting for tumor and normal tissue samples. Our quantification data showed that, compared to normal tissues, TTP protein levels in breast cancer tissues were significantly reduced (Figure 1D). Meanwhile, we performed immunocytochemistry to see the protein expression of TTP in individual tumors and normal cells. We found that high protein levels of TTP were present in normal tissues (positive cells 
displaying brown particles), while DCIS and IDC breast tumor tissues exhibited undetectable TTP levels (Figure 1E).

\section{Resveratrol inhibits MCF-7 cell proliferation}

Resveratrol can inhibit the development of various human malignant tumors including breast tumors. Different research groups have demonstrated the inhibitory effect of resveratrol on the proliferation of breast cancer cell lines including MCF-7 (Banerjee et al., 2002; Degner et al., 2007). To obtain a working concentration of resveratrol in our experimental condition, we performed an MTT assay on the growth of MCF-7 cells over 7 days with different concentrations of resveratrol $(0,10,20$, and $40 \mu \mathrm{M})$. We found that $20 \mu \mathrm{M}$ resveratrol caused a larger reduction in MCF-7 cell proliferation rate than $10 \mu \mathrm{M}$. However, there was little difference between 20 and $40 \mu \mathrm{M}$ resveratrol treatment, suggesting that $20 \mu \mathrm{M}$ was the saturating concentration (Figure 2A).

A

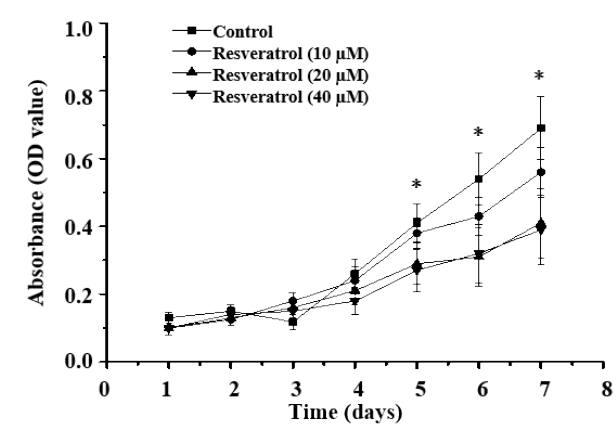

C

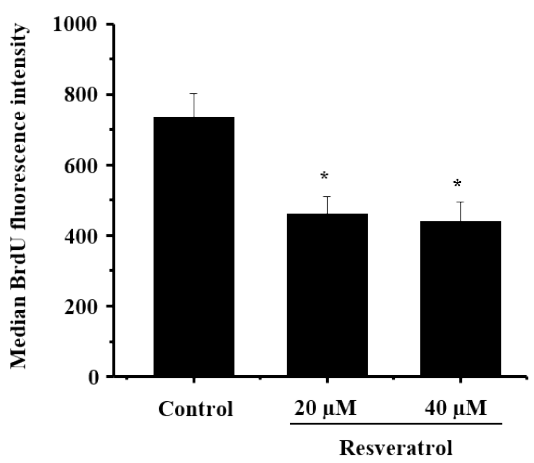

B
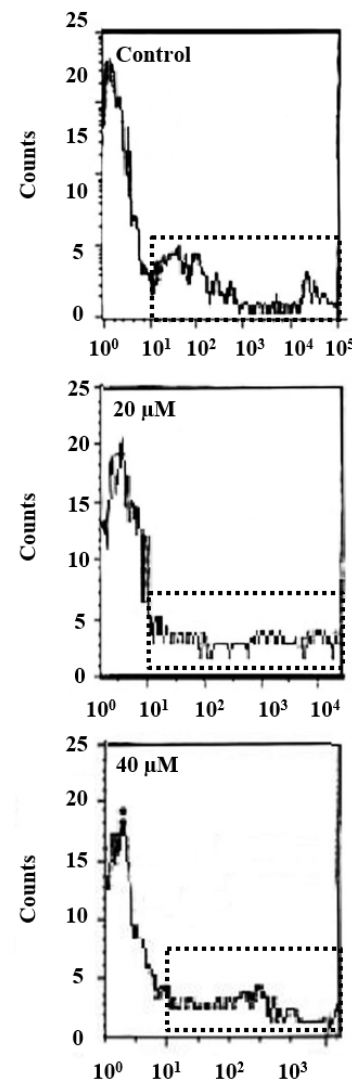

Figure 2. Inhibitory effect of resveratrol on MCF-7 cell proliferation. A. MTT assay showing the effects of different concentrations of resveratrol $(0,10,20$, and $40 \mu \mathrm{M})$ on MCF-7 cell growth over 7 days. $* \mathrm{P}<0.05$. B. Flow cytometry data of MCF-7 cells treated with different concentrations of resveratrol $(0,20$, and $40 \mu \mathrm{M})$. The rectangle region marked by dashed lines was selected for qualification. C. Comparison between the untreated and resveratrol-treated groups in flow cytometry analysis $(\mathrm{N}=3) . * \mathrm{P}<0.05$. 
In parallel, we performed flow cytometry to examine the inhibiting effect of different concentrations of resveratrol $(0,20$, and $40 \mu \mathrm{M})$ on MCF-7 cell proliferation. Consistently, we found BrdU mean fluorescence intensity in resveratrol-treated cells $(20$ and $40 \mu \mathrm{M})$ was significantly lower than that in the control cells (Figure 2B and C).

\section{Effects of resveratrol on TTP expression and downstream pathways in MCF-7 cells}

It has been shown that TTP expression can be regulated through NF-kB, p38MAPK, STATs, and other signaling pathways (Brook et al., 2006; Joe et al., 2011). Resveratrol has been confirmed to play effective roles in the same signaling pathways (Bhardwaj et al., 2007). Therefore, it is highly possible that resveratrol acts against cancers via TTP mediation. To investigate whether TTP is a mediator or functions independently of the effect of resveratrol on tumor cell proliferation, we treated MCF-7 cells with different concentrations of resveratrol $(0,10,20$, and $40 \mu \mathrm{M})$ for $6 \mathrm{~h}$ and examined the mRNA and protein levels of TTP in the treated cells. We found that all three concentrations of resveratrol significantly increased TTP mRNA expression with a slight difference between 20 and $40 \mu \mathrm{M}$ (Figure 3A). Resveratrol (20 and $40 \mu \mathrm{M})$ significantly increased protein expression of TTP while $10 \mu \mathrm{M}$ resveratrol failed to cause a significant increase (Figure 3B), suggesting that a small increase in mRNA level was not enough to cause a significant increase in protein expression.
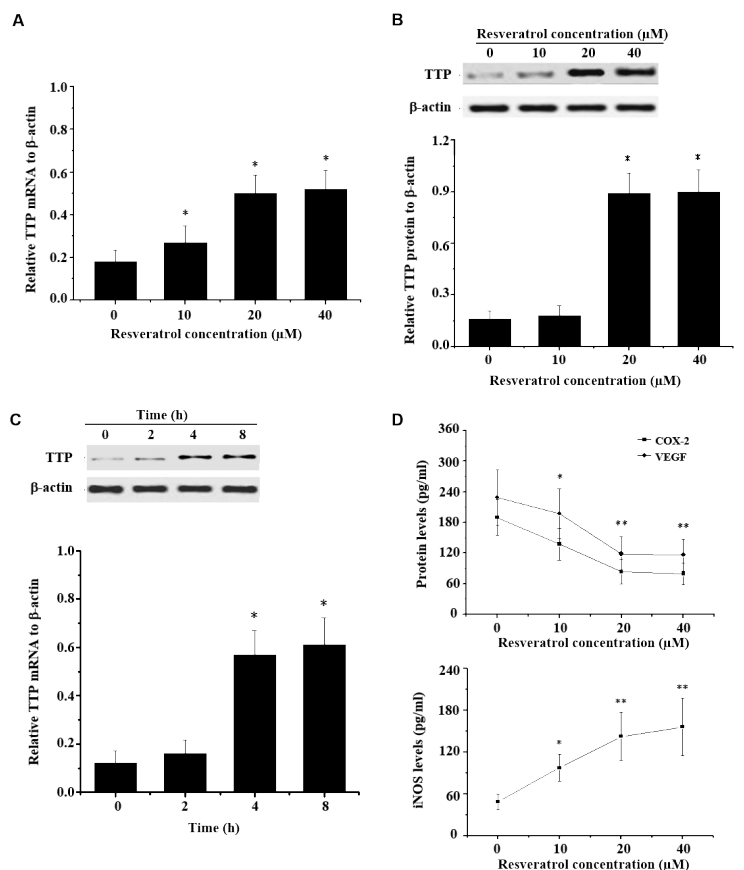

Figure 3. Effects of resveratrol on TTP expression and downstream pathways in MCF-7 cells. A. qRT-PCR assay showing the effect of different concentrations of resveratrol $(0,10,20$, and $40 \mu \mathrm{M})$ on TTP mRNA expression levels (N $=3) . * \mathrm{P}<0.05$. B. Western blotting showing the effect of different concentrations of resveratrol $(0,10,20$, and $40 \mu \mathrm{M})$ on TTP protein expression levels $(\mathrm{N}=3)$. $* \mathrm{P}<0.05$. C. Western blotting showing resveratrol promotes TTP protein expression in a time-dependent manner $(\mathrm{N}=3) . * \mathrm{P}<0.05$. D. ELISA showing the effects of different concentrations of resveratrol $(0,10,20$, and $40 \mu \mathrm{M})$ on the protein levels of COX-2, VEGF, and iNOS $(\mathrm{N}=3) .{ }^{*} \mathrm{P}<0.05,{ }^{*} \mathrm{P}<0.01$. 
In addition, we investigated the time course for the effect of resveratrol on TTP expression by examining the TTP expression levels at different time points $(0,2,4$, or $8 \mathrm{~h})$ during treatment with $20 \mu \mathrm{M}$ resveratrol. Our results showed that resveratrol significantly upregulated TTP expression at 4 and $8 \mathrm{~h}$, while the effect was not yet significant at $2 \mathrm{~h}$ (Figure 3C).

Together, our data suggested that resveratrol upregulates TTP expression in a doseand time-dependent manner.

COX-2, VEGF, and iNOS are downstream effectors of TTP in human tumors and cell lines. To investigate whether resveratrol has a regulating effect on these downstream effectors (Fechir et al., 2005; Lee et al., 2010; Cha et al., 2011), we treated MCF-7 cells with different concentrations of resveratrol $(0,10,20$, and $40 \mu \mathrm{M})$ for $6 \mathrm{~h}$ and performed ELISA to obtain the protein levels of COX-2, VEGF, and iNOS. Our results showed that, in a dosedependent manner, resveratrol significantly decreased the expression of COX-2 and VEGF while increasing the expression of iNOS (Figure 3D), suggesting that TTP may mediate the anticancer effects of resveratrol by regulating COX-2, VEGF, and iNOS expressions.

\section{RNA interference confirms the role of TTP in the anticancer effects of resveratrol in MCF-7 cells}

To further investigate if TTP is a mediator of the effects of resveratrol on cell proliferation and downstream pathways, we performed RNA interference on MCF-7 cells treated with 0 or $20 \mu \mathrm{M}$ resveratrol. We used Lipofectamine 2000 to transfect $10 \mathrm{nM}$ TTP or control siRNA into MCF-7 cells and examined the expression levels of TTP by western blotting $48 \mathrm{~h}$ following transfection. The results showed that TTP siRNA effectively decreased the protein levels of TTP (Figure 4A).

To investigate whether the TTP siRNA can rescue the resveratrol-induced reduction of COX-2 and VEGF expression, we combined the siRNA treatment and resveratrol treatment and performed ELISA to examine the changes in the levels of COX-2 and VEGF in MCF-7 cells following the treatments. We measured the expression reduction rate by dividing the protein level of the resveratrol-treated cells by that of DMSO-treated cells. The higher the rate, the less the protein expression was changed. We found that the COX-2 and VEGF expression rate in TTP siRNA-treated cells were 86.5 and $86.9 \%$, respectively. In contrast, the rates of control siRNA-treated cells were 44.3 and $51.8 \%$, respectively. Our statistical analysis showed that the differences in both expression rates between TTP and control siRNA treatments were significant (Figure 4B and C), indicating that TTP siRNA significantly rescued the expression reduction induced by resveratrol.

To investigate whether TTP regulates COX-2 expression by promoting mRNA degradation, we measured COX-2 mRNA degradation kinetics in MCF-7 cells with four different treatments: control siRNA/DMSO, control siRNA/20 $\mu \mathrm{M}$ resveratrol, TTP siRNA/ DMSO, and TTP siRNA/20 $\mu \mathrm{M}$ resveratrol. We found that, compared to the control siRNA/ resveratrol treatment, TTP siRNA/resveratrol treatment significantly reduced the COX-2 mRNA degradation rate starting from 40 min (Figure 4D), suggesting that TTP inhibits mRNA expression by promoting its mRNA degradation.

Lastly, to further demonstrate that the effect of resveratrol on tumor cell proliferation is mediated by TTP, we performed an MTT assay on MCF-7 cells with the four treatments as above and plotted the 7-day growth curves. We calculated the ratio of OD values of resveratroltreated to DMSO-treated at individual time points. We found that, at day 7 , resveratrol 
inhibited the MCF-7 cell proliferation rate by $16.4 \%$ in the TTP siRNA treatment group, while it inhibited the MCF-7 cell proliferation rate by $39.4 \%$ in the control siRNA treatment group. Our statistical analysis showed that the difference between the two rates was significant (Figure 4E), demonstrating that TTP mediates the anti-proliferative effect of resveratrol.

A
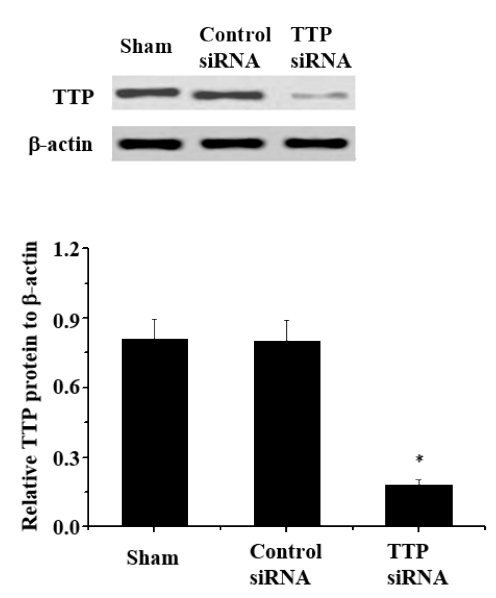

D

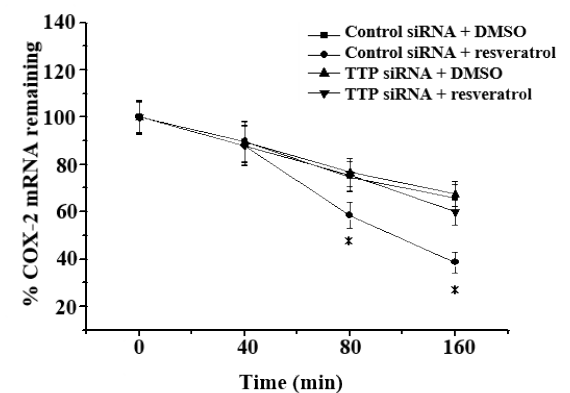

B

C

E
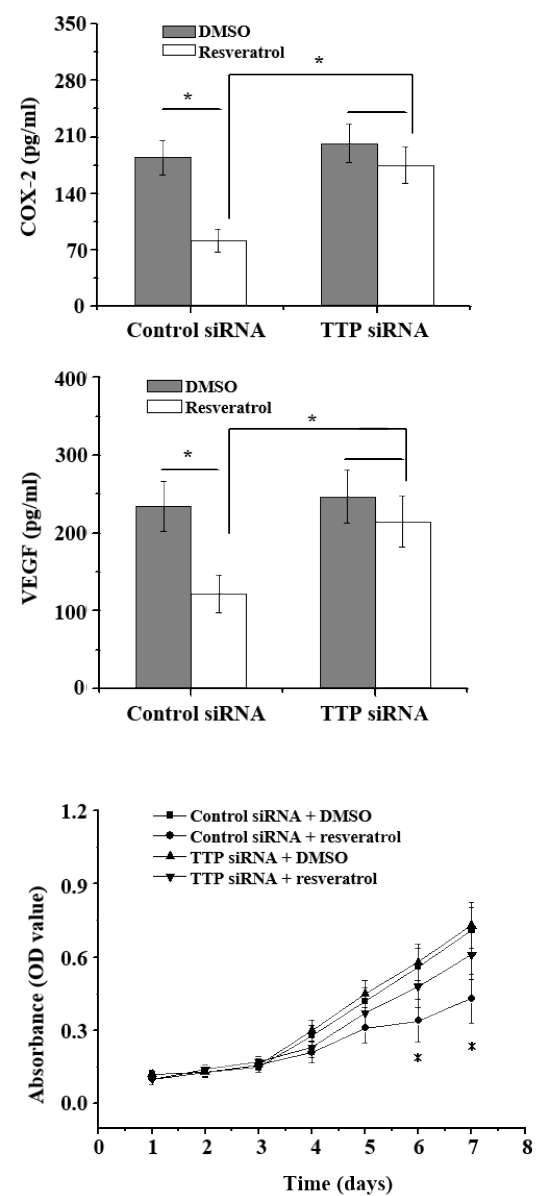

Figure 4. Role of TTP in the anticancer effects of resveratrol in MCF-7 cells. A. Western blotting showing the effect of TTP and control siRNA on the protein levels of TTP $(\mathrm{N}=3)$. $* \mathrm{P}<0.05$. B. ELISA showing TTP siRNA inhibited the downregulating effect of resveratrol on COX-2 expression $(\mathrm{N}=3)$. $* \mathrm{P}<0.05$. C. ELISA showing TTP siRNA inhibited the downregulating effect of resveratrol on VEGF expression $(\mathrm{N}=3)$. D. qRT-PCR measured mRNA degradation rate of COX-2 across $160 \mathrm{~min}$, showing TTP siRNA rescued resveratrol-induced reduction of COX-2 expression $(\mathrm{N}=3)$. $* \mathrm{P}<0.05$. E. MTT assay on cell growth over 7 days, showing TTP siRNA rescued resveratrol-induced decrease of cell proliferation $(\mathrm{N}=3) . * \mathrm{P}<0.05$.

\section{Role of TTP in the anticancer effects of resveratrol confirmed by using a different breast cancer cell line MDA-MB-231}

To investigate if the regulating role of TTP on the effects of resveratrol is specific to MCF-7 cells, we performed similar experiments with a different breast cancer cell line, MDA- 
MB-231. First, we performed an MTT assay on the growth of MDA-MB-231 cells. We found that resveratrol reduced the MDA-MB-231 cell proliferation rate (Figure 5A). Second, we treated MDA-MB-231 cells with different concentrations of resveratrol $(0,10,20$, and $40 \mu \mathrm{M})$ for $6 \mathrm{~h}$ and found that resveratrol significantly increased TTP mRNA expression (Figure 5B) and protein expression (Figure 5C). Third, we looked at the time course for the treatment of MDAMB-231 cells with $20 \mu \mathrm{M}$ resveratrol and found that resveratrol significantly upregulated TTP expression starting at 4 days (Figure 5D). Last, we examined the change in the expression of COX-2, VEGF, and iNOS by resveratrol and found that resveratrol significantly decreased the expression of COX-2 and VEGF while increasing the expression of iNOS in MDA-MB-231 cells (Figure 5E). In summary, we demonstrated that resveratrol can increase TTP expression and further alter the expression of TTP downstream effectors using a different cell line.

A
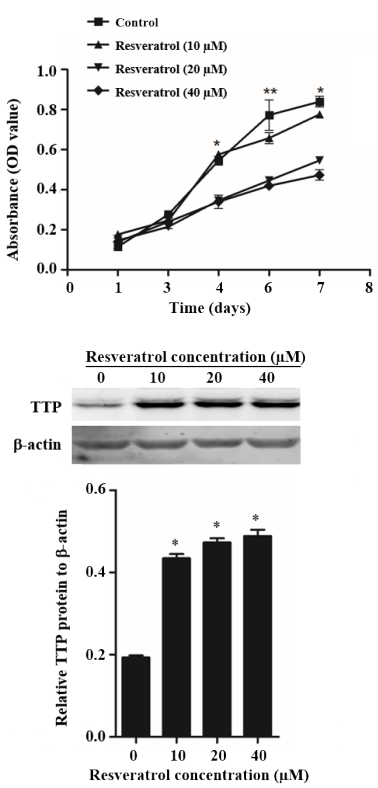

E

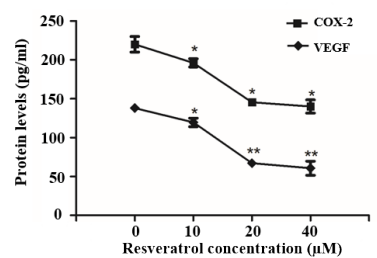

B

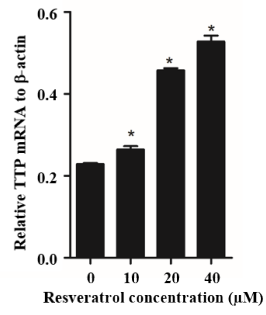

D
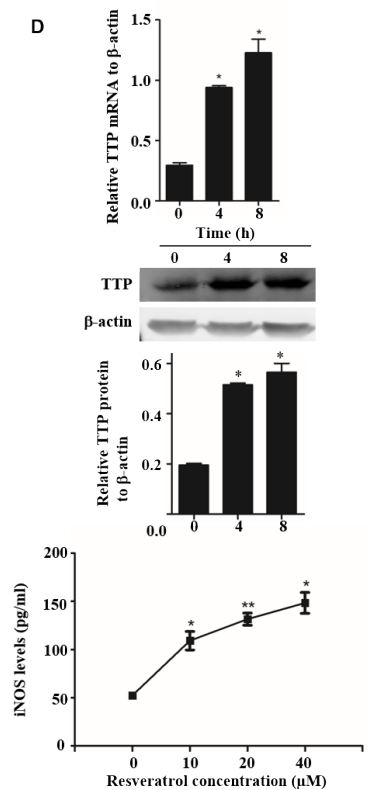

Figure 5. Effects of resveratrol on cell proliferation, TTP expression, and downstream pathways in MDA-MB-231 cells. A. MTT assay showing the effects of different concentrations of resveratrol $(0,10,20$, and $40 \mu \mathrm{M})$ on MDAMB-231 cell growth over 7 days. B. qRT-PCR assay showing the effect of different concentrations of resveratrol $(0,10,20$, and $40 \mu \mathrm{M})$ on TTP mRNA expression levels. C. Western blotting showing the effect of different concentrations of resveratrol $(0,10,20$, and $40 \mu \mathrm{M})$ on TTP protein expression levels. D. qRT-PCR and western blotting showing resveratrol promotes TTP protein expression in a time-dependent manner. E. ELISA showing the effects of different concentrations of resveratrol $(0,10,20$, and $40 \mu \mathrm{M})$ on the protein expression levels of COX-2, VEGF, and iNOS. ${ }^{*} \mathrm{P}<0.05,{ }^{*} * \mathrm{P}<0.01$. 
We further performed RNA interference on MDA-MB-231 cells to demonstrate the mediation of TTP in the anticancer effects of resveratrol. First, we found that TTP siRNA effectively decreased the protein levels of TTP (Figure 6A). Second, we measured the expression levels of COX-2 and VEGF in the cells following the treatment of siRNA with resveratrol/DMSO and found that there was a significant difference between the control siRNA and TTP siRNA treatments on both COX-2 and VEGF expression (Figure 6B and C). Third, we measured COX-2 mRNA degradation kinetics in MDA-MB-231 cells and found that, compared to the control siRNA/resveratrol treatment, TTP siRNA/resveratrol treatment significantly reduced the COX-2 mRNA degradation rate starting at $40 \mathrm{~min}$ (Figure 6D). Last, we performed an MTT assay on MDA-MB-231 cells with the treatments and demonstrated that TTP mediates the anti-proliferative effect of resveratrol (Figure 6E).

A
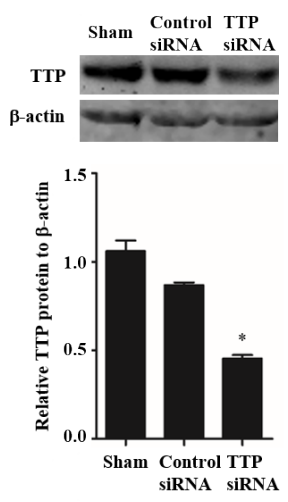

D

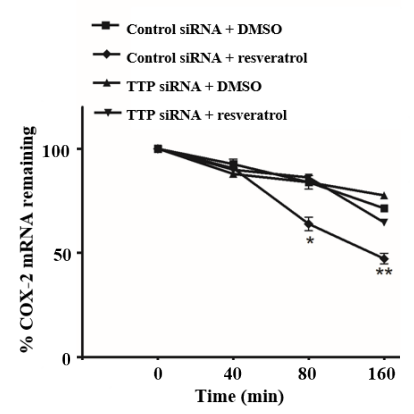

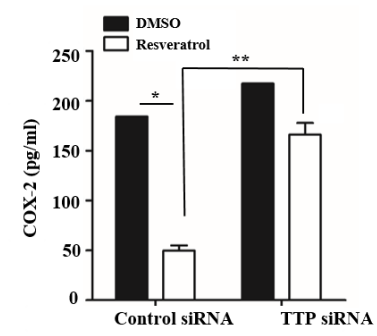

C

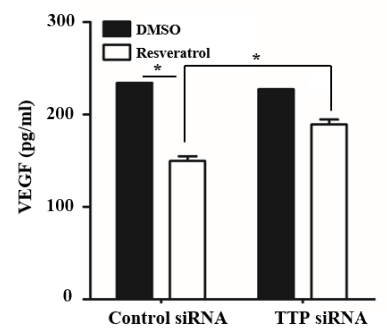

E

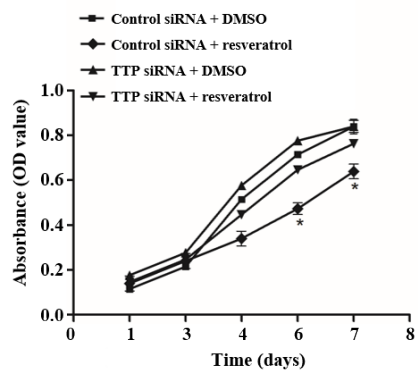

Figure 6. Role of TTP in the anticancer effects of resveratrol in MDA-MB-231 cells. A. Western blotting showing the effect of TTP and control siRNA on the protein expression levels of TTP. B. ELISA showing TTP siRNA treatment inhibited the downregulating effect of resveratrol on COX-2 expression. C. ELISA showing TTP siRNA treatment inhibited the downregulating effect of resveratrol on VEGF expression. D. qRT-PCR measured mRNA degradation rate of COX-2 across $160 \mathrm{~min}$, showing TTP siRNA treatment rescued resveratrol-induced reduction of COX-2 expression. E. MTT assay on cell growth over 7 days, showing TTP siRNA treatment rescued resveratrolinduced decrease of cell proliferation. $* \mathrm{P}<0.05,{ }^{*} \mathrm{P}<0.01$. 
Taken together, the data obtained from the MDA-MB-231 cells were similar to those from MCF-7 cells, confirming that the regulating role of TTP on the effects of resveratrol is not specific to MCF-7 cells.

\section{DISCUSSION}

Various cytokines and cytokine-producing enzymes play key roles in the cell proliferation of malignant tumors. COX-2 and VEGF are overexpressed in a variety of tumors including breast cancers and promote tumor cell proliferation (Falandry et al., 2009), suggesting that downregulating COX-2 and VEGF expression is very important in cancer prevention and treatment. TTP can reduce the protein expression of a variety of cytokines by promoting mRNA degradation. Upregulation of TTP is a new method of cancer prevention (Griseri et al., 2011; Kim et al., 2012). TTP expression can be regulated through NF-kB, p38MAPK, STATs and other signaling pathways (Brook et al., 2006; Joe et al., 2011). Resveratrol has been confirmed to have effective actions on these signaling pathways (Bhardwaj et al., 2007). Therefore, it is worthwhile to investigate whether resveratrol acts against cancers through the mediation of TTP. Here, we show that resveratrol can inhibit TTP expression in a doseand time-dependent manner (Figure 3A-C). Most importantly, we found that the effects of resveratrol on cell proliferation as well as the expression of VEGF and COX-2 are notably inhibited by knocking down TTP expression by RNA interference, demonstrating that TTP is a potential key mediator for the anticancer effects of resveratrol (Figure 4). To our knowledge, this is the first evidence to date regarding the role of TTP in the anticancer effects of resveratrol.

By regulating mRNA degradation, mRNA binding proteins play a key role in the regulation of gene expression in mammals. Here, we examined the expression levels of three common mRNA binding proteins including AUF1, HuR, and TTP in two different breast cancer types (DCIS and IDC). Consistent with the findings of Brennan et al. (2009), we show that both mRNA and protein levels of TTP are substantially decreased in DCIS and IDC breast tumors relative to normal pericarcinous tissues (Figure 1). In contrast, HuR and AUF1 expressions are not significantly altered in these tumors relative to normal controls. Moreover, by studying a different human population, we demonstrate that the expression changes of these three genes in breast tumors do not differ between Western and Chinese populations, providing the first evidence for using suppressed TTP expression as a negative prognostic indicator of breast cancers in the Chinese population.

The investigation of the anticancer mechanism of resveratrol has become a popular biomedical research topic. It is believed that, besides its unique anti-inflammatory and antioxidant effects, its inhibitory effects on cell proliferation, apoptosis, and invasion are its key actions against cancers (De Leo et al., 2011). In gastric cancers, Holian et al. (2002) found that resveratrol promotes iNOS activity by increasing the NO level, thereby inhibiting the proliferation of gastric cancer cells. In prostate cancers, resveratrol has been found to inhibit androgen-stimulated proliferation of $\mathrm{LNCaP}, \mathrm{PC} 3$, and Du145 prostate cancer cell lines by inhibiting the expression of the androgen receptor (Kai and Levenson, 2011). In colon cancers, resveratrol has been found to inhibit cell proliferation of colon cancer cell lines by inhibiting the formation of CD1 and the cyclin-dependent kinase 4 complex (Wolter et al., 2001). In renal cancers, Yang et al. (2011) found that resveratrol can inhibit the expression of VEGF, thereby inhibiting the proliferation of renal cancer cell lines. In breast cancers, resveratrol and its analogues have been found to inhibit the proliferation of breast cancer cell 
lines by inhibiting nuclear factor-kappaB and COX-2 expression (Banerjee et al., 2002). In this study, we have proven that resveratrol can inhibit the proliferation of MCF-7 and MDAMB-231 breast cancer cell lines (Figure 2). We found that, in both types of cells, resveratrol can inhibit VEGF and COX-2 expression (Figure 3D). Further, we show that the resveratrolinduced degradation of COX-2 mRNA is notably inhibited by TTP knock down, suggesting that TTP mediates the inhibiting effect of resveratrol on the expression of COX-2 and other downstream effectors by increasing the rate of mRNA degradation of these genes (Figure 4D). Meanwhile, we found that resveratrol can significantly increase the level of iNOS, suggesting that high levels of iNOS expression may be one of the mechanisms through which resveratrol inhibits tumor development. However, how resveratrol upregulates iNOS expression needs to be further investigated.

In addition, the p38MAPK signaling pathway can significantly promote TTP phosphorylation and TTP relocation from the nucleus into the cytoplasm, thereby stabilizing its expression and function (Brook et al., 2006). In breast cancer cell lines, resveratrol has been found to engage in p38MAPK signal pathway activation (De Amicis et al., 2011). Determining whether resveratrol upregulates TTP expression through the p38MAPK signaling pathway requires further investigation.

We conclude that resveratrol inhibits the proliferation of breast cancer cells by upregulating TTP expression and further by altering the expressions of downstream effectors of TTP including VEGF, COX-2, and iNOS. The novel finding that TTP mediates the anticancer effects of resveratrol strengthens our understanding of the mechanism through which resveratrol acts against cancers.

\section{Conflicts of interest}

The authors declare $t$ they have no conflict of interests.

\section{REFERENCES}

Ahmad S, Ahmad A, Schneider KB and White CW (2006). Cholesterol interferes with the MTT assay in human epithelial-like (A549) and endothelial (HLMVE and HCAE) cells. Int. J. Toxicol. 25: 17-23. http://dx.doi. org/10.1080/10915810500488361

Banerjee S, Bueso-Ramos C and Aggarwal BB (2002). Suppression of 7,12-dimethylbenz(a)anthracene-induced mammary carcinogenesis in rats by resveratrol: role of nuclear factor-kappaB, cyclooxygenase 2, and matrix metalloprotease 9. Cancer Res. 62: 4945-4954

Bhardwaj A, Sethi G, Vadhan-Raj S, Bueso-Ramos C, et al. (2007). Resveratrol inhibits proliferation, induces apoptosis, and overcomes chemoresistance through down-regulation of STAT3 and nuclear factor-kappaB-regulated antiapoptotic and cell survival gene products in human multiple myeloma cells. Blood 109: 2293-2302. http://dx.doi. org/10.1182/blood-2006-02-003988

Bishayee A (2009). Cancer prevention and treatment with resveratrol: from rodent studies to clinical trials. Cancer Prev. Res. 2: 409-418. http://dx.doi.org/10.1158/1940-6207.CAPR-08-0160

Brennan SE, Kuwano Y, Alkharouf N, Blackshear PJ, et al. (2009). The mRNA-destabilizing protein tristetraprolin is suppressed in many cancers, altering tumorigenic phenotypes and patient prognosis. Cancer Res. 69: 5168-5176. http://dx.doi.org/10.1158/0008-5472.CAN-08-4238

Brook M, Tchen CR, Santalucia T, McIlrath J, et al. (2006). Posttranslational regulation of tristetraprolin subcellular localization and protein stability by p38 mitogen-activated protein kinase and extracellular signal-regulated kinase pathways. Mol. Cell. Biol. 26: 2408-2418. http://dx.doi.org/10.1128/MCB.26.6.2408-2418.2006

Cao H, Kelly MA, Kari F, Dawson HD, et al. (2007). Green tea increases anti-inflammatory tristetraprolin and decreases proinflammatory tumor necrosis factor mRNA levels in rats. J. Inflamm. (Lond.) 4: 1. http://dx.doi.org/10.1186/1476-9255-4-1 
Cao H, Urban JF, Jr. and Anderson RA (2008). Insulin increases tristetraprolin and decreases VEGF gene expression in mouse 3T3-L1 adipocytes. Obesity (Silver Spring) 16: 1208-1218. http://dx.doi.org/10.1038/oby.2008.65

Cha HJ, Lee HH, Chae SW, Cho WJ, et al. (2011). Tristetraprolin downregulates the expression of both VEGF and COX-2 in human colon cancer. Hepatogastroenterology 58: 790-795.

De Amicis F, Giordano F, Vivacqua A, Pellegrino M, et al. (2011). Resveratrol, through NF-Y/p53/Sin3/HDAC1 complex phosphorylation, inhibits estrogen receptor alpha gene expression via p38MAPK/CK2 signaling in human breast cancer cells. FASEB J. 25: 3695-3707. http://dx.doi.org/10.1096/fj.10-178871

De Leo A, Arena G, Stecca C, Raciti M, et al. (2011). Resveratrol inhibits proliferation and survival of Epstein Barr virusinfected Burkitt's lymphoma cells depending on viral latency program. Mol. Cancer Res. 9: 1346-1355. http://dx.doi. org/10.1158/1541-7786.MCR-11-0145

Degner SC, Kemp MQ, Hockings JK and Romagnolo DF (2007). Cyclooxygenase-2 promoter activation by the aromatic hydrocarbon receptor in breast cancer mcf-7 cells: repressive effects of conjugated linoleic acid. Nutr. Cancer 59: 248-257. http://dx.doi.org/10.1080/01635580701485585

Falandry C, Canney PA, Freyer G and Dirix LY (2009). Role of combination therapy with aromatase and cyclooxygenase-2 inhibitors in patients with metastatic breast cancer. Ann. Oncol. 20: 615-620. http://dx.doi.org/10.1093/annonc/mdn693

Falandry C, Brain E, Bonnefoy M, Mefti F, et al. (2011). Impact of geriatric vulnerability parameters on pegylated liposomal doxorubicin (PLD) tolerance and outcome in elderly patients with metastatic breast cancer: Results of the DOGMES multicenter phase II GINECO trial. J. Clin. Oncol. 29: e19740.

Fechir M, Linker K, Pautz A, Hubrich T, et al. (2005). Tristetraprolin regulates the expression of the human inducible nitric-oxide synthase gene. Mol. Pharmacol. 67: 2148-2161. http://dx.doi.org/10.1124/mol.104.008763

Griseri P, Bourcier C, Hieblot C, Essafi-Benkhadir K, et al. (2011). A synonymous polymorphism of the Tristetraprolin (TTP) gene, an AU-rich mRNA-binding protein, affects translation efficiency and response to Herceptin treatment in breast cancer patients. Hum. Mol. Genet. 20: 4556-4568. http://dx.doi.org/10.1093/hmg/ddr390

Hanahan D and Weinberg RA (2011). Hallmarks of cancer: the next generation. Cell 144: 646-674. http://dx.doi. org/10.1016/j.cell.2011.02.013

Harada K, Supriatno, Kawaguchi S, Tomitaro O, et al. (2004). Overexpression of iNOS gene suppresses the tumorigenicity and metastasis of oral cancer cells. In Vivo 18: 449-455.

Hitti E, Iakovleva T, Brook M, Deppenmeier S, et al. (2006). Mitogen-activated protein kinase-activated protein kinase 2 regulates tumor necrosis factor mRNA stability and translation mainly by altering tristetraprolin expression, stability, and binding to adenine/uridine-rich element. Mol. Cell. Biol. 26: 2399-2407. http://dx.doi.org/10.1128/ MCB.26.6.2399-2407.2006

Holian O, Wahid S, Atten MJ and Attar BM (2002). Inhibition of gastric cancer cell proliferation by resveratrol: role of nitric oxide. Am. J. Physiol. Gastrointest. Liver Physiol. 282: G809-G816. http://dx.doi.org/10.1152/ajpgi.00193.2001

Hong CW and Zeng Q (2012). Awaiting a new era of cancer immunotherapy. Cancer Res. 72: 3715-3719. http://dx.doi. org/10.1158/0008-5472.CAN-12-0063

Joe Y, Kim HJ, Kim S, Chung J, et al. (2011). Tristetraprolin mediates anti-inflammatory effects of nicotine in lipopolysaccharide-stimulated macrophages. J. Biol. Chem. 286: 24735-24742. http://dx.doi.org/10.1074/jbc. M110.204859

Kai L and Levenson AS (2011). Combination of resveratrol and antiandrogen flutamide has synergistic effect on androgen receptor inhibition in prostate cancer cells. Anticancer Res. 31: 3323-3330.

Kim CW, Vo MT, Kim HK, Lee HH, et al. (2012). Ectopic over-expression of tristetraprolin in human cancer cells promotes biogenesis of let-7 by down-regulation of Lin28. Nucleic Acids Res. 40: 3856-3869. http://dx.doi. org/10.1093/nar/gkr1302

Lee HH, Son YJ, Lee WH, Park YW, et al. (2010). Tristetraprolin regulates expression of VEGF and tumorigenesis in human colon cancer. Int. J. Cancer 126: 1817-1827.

Maquat LE (2005). Nonsense-mediated mRNA decay in mammals. J. Cell Sci. 118: 1773-1776. http://dx.doi.org/10.1242/ jes. 01701

Melanson BD, Bose R, Hamill JD, Marcellus KA, et al. (2011). The role of mRNA decay in p53-induced gene expression. RNA 17: 2222-2234. http://dx.doi.org/10.1261/rna.030122.111

Ogilvie RL, Abelson M, Hau HH, Vlasova I, et al. (2005). Tristetraprolin down-regulates IL-2 gene expression through AU-rich element-mediated mRNA decay. J. Immunol. 174: 953-961. http://dx.doi.org/10.4049/jimmunol.174.2.953

Patino WD, Kang JG, Matoba S, Mian OY, et al. (2006). Atherosclerotic plaque macrophage transcriptional regulators are expressed in blood and modulated by tristetraprolin. Circ. Res. 98: 1282-1289. http://dx.doi.org/10.1161/01. RES.0000222284.48288.28

Qiu LQ, Stumpo DJ and Blackshear PJ (2012). Myeloid-specific tristetraprolin deficiency in mice results in extreme 
lipopolysaccharide sensitivity in an otherwise minimal phenotype. J. Immunol. 188: 5150-5159. http://dx.doi. org/10.4049/jimmunol.1103700

Sanduja S, Blanco FF, Young LE, Kaza V, et al. (2012). The role of tristetraprolin in cancer and inflammation. Front. Biosci. (Landmark Ed.) 17: 174-188. http://dx.doi.org/10.2741/3920

Shchors K, Yehiely F, Kular RK, Kotlo KU, et al. (2002). Cell death inhibiting RNA (CDIR) derived from a 3'-untranslated region binds AUF1 and heat shock protein 27. J. Biol. Chem. 277: 47061-47072. http://dx.doi.org/10.1074/jbc. M202272200

Subramanian L, Youssef S, Bhattacharya S, Kenealey J, et al. (2010). Resveratrol: challenges in translation to the clinic--a critical discussion. Clin. Cancer Res. 16: 5942-5948. http://dx.doi.org/10.1158/1078-0432.CCR-10-1486

Takahashi M, Kitahashi T, Ishigamori R, Mutoh M, et al. (2008). Increased expression of inducible nitric oxide synthase (iNOS) in N-nitrosobis(2-oxopropyl)amine-induced hamster pancreatic carcinogenesis and prevention of cancer development by ONO-1714, an iNOS inhibitor. Carcinogenesis 29: 1608-1613. http://dx.doi.org/10.1093/carcin/bgn152

Tan DS, Marchiò C and Reis-Filho JS (2008). Hereditary breast cancer: from molecular pathology to tailored therapies. $J$. Clin. Pathol. 61: 1073-1082. http://dx.doi.org/10.1136/jcp.2008.057950

Wolter F, Akoglu B, Clausnitzer A and Stein J (2001). Downregulation of the cyclin D1/Cdk4 complex occurs during resveratrol-induced cell cycle arrest in colon cancer cell lines. J. Nutr. 131: 2197-2203.

Yang R, Zhang H and Zhu L (2011). Inhibitory effect of resveratrol on the expression of the VEGF gene and proliferation in renal cancer cells. Mol. Med. Rep. 4: 981-983.

Yin K, Deng X, Mo ZC, Zhao GJ, et al. (2011). Tristetraprolin-dependent post-transcriptional regulation of inflammatory cytokine mRNA expression by apolipoprotein A-I: role of ATP-binding membrane cassette transporter A1 and signal transducer and activator of transcription 3. J. Biol. Chem. 286: 13834-13845. http://dx.doi.org/10.1074/jbc. $\underline{\mathrm{M} 110.202275}$

Yin S, Deng W, Zheng H, Zhang Z, et al. (2009). Evidence that the nonsense-mediated mRNA decay pathway participates in X chromosome dosage compensation in mammals. Biochem. Biophys. Res. Commun. 383: 378-382. http://dx.doi. org/10.1016/j.bbrc.2009.04.021

Young LE, Moore AE, Sokol L, Meisner-Kober N, et al. (2012). The mRNA stability factor HuR inhibits microRNA-16 targeting of COX-2. Mol. Cancer Res. 10: 167-180. http://dx.doi.org/10.1158/1541-7786.MCR-11-0337

Zhang Q, Tang X, Lu QY, Zhang ZF, et al. (2005). Resveratrol inhibits hypoxia-induced accumulation of hypoxiainducible factor-1alpha and VEGF expression in human tongue squamous cell carcinoma and hepatoma cells. Mol. Cancer Ther. 4: 1465-1474. http://dx.doi.org/10.1158/1535-7163.MCT-05-0198 